\title{
Beneficial effects of astragaloside IV against angiotensin II-induced mitochondrial dysfunction in rat vascular smooth muscle cells
}

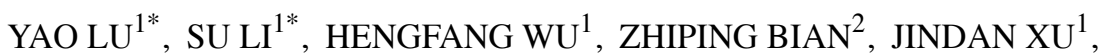 \\ CHUNRONG GU ${ }^{2}$, XIANGJIAN $\mathrm{CHEN}^{2}$ and DI YANG ${ }^{1}$ \\ ${ }^{1}$ Department of Cardiology, and ${ }^{2}$ Research Institute of Cardiovascular Disease, \\ The First Affiliated Hospital of Nanjing Medical University, Nanjing, Jiangsu 210029, P.R. China
}

Received March 1, 2015; Accepted September 8, 2015

DOI: $10.3892 /$ ijmm.2015.2345

\begin{abstract}
Angiotensin II (Ang II)-induced mitochondrial dysfunction is a prominent characteristic of the majority of cardiovascular diseases. Astragaloside IV (As-IV), the major active ingredient of Astragalus membranaceus (Fisch.) Bge. (a traditional Chinese herbal medicine), possesses antioxidant properties. The present study was carried out to examine whether As-IV can reverse Ang II-induced mitochondrial dysfunction in vascular smooth muscle cells (VSMCs) and to elucidate the underlying molecular mechanisms. Cultured rat aortic VSMCs treated with Ang II $(1 \mu \mathrm{M})$ for $24 \mathrm{~h}$ exhibited mitochondrial dysfunction, including a decrease in mitochondrial oxygen consumption rates (OCRs), adenosine triphosphate (ATP) production and mitochondrial DNA (mtDNA) levels, as well as the disruption of mitochondrial structural integrity. Following treatment with Ang II, As-IV $(50 \mu \mathrm{g} / \mathrm{ml})$ was added to the culture medium followed by incubation for a further $24 \mathrm{~h}$. The administration of As-IV significantly increased the mitochondrial OCRs, ATP production and the mtDNA levels, and reversed the mitochondrial morphological changes which occurred in the VSMCs. Treatment with As-IV also reversed the Ang II-induced increase in the production of reactive oxygen species (ROS), the increase in NADPH oxidase and xanthine oxidase activity, as well as the decrease in mitochon-
\end{abstract}

Correspondence to: Dr Xiangjian Chen, Research Institute of Cardiovascular Disease, The First Affiliated Hospital of Nanjing Medical University, 300 Guangzhou Road, Nanjing, Jiangsu 210029, P.R. China

E-mail: chenxiangjian@njmu.edu.cn

Professor Di Yang, Department of Cardiology, The First Affiliated Hospital of Nanjing Medical University, 300 Guangzhou Road, Nanjing, Jiangsu 210029, P.R. China

E-mail: diyang@njmu.edu.cn

*Contributed equally

Key words: astragaloside IV, mitochondrial dysfunction, ROS, smooth muscle cells drial membrane potential $(\Delta \Psi \mathrm{m})$ and manganese superoxide dismutase (Mn-SOD) activity. Furthermore, treatment with As-IV led to an increase in the mRNA expression of peroxisome proliferator-activated receptor-gamma coactivator-1 $\alpha$ (PGC$1 \alpha$ ) and mitochondrial transcription factor A (Tfam), and in the protein expression of PGC-1 $\alpha$, parkin and dynamin 1-like protein 1 (Drp1) in the VSMCs. These results indicate that As-IV exerts beneficial effects on Ang II-induced mitochondrial dysfunction in rat VSMCs and that these effects are mediated through the inhibition of ROS overproduction, as well as the promotion of mitochondrial autophagy and mitochondrial biogenesis. These data demonstrate the antioxidant properties of As-IV.

\section{Introduction}

Mitochondria are known as cellular powerhouses due to their ability to generate adenosine triphosphate (ATP) for use in metabolic processes. These intracellular organelles of bacterial origin, are important for bioenergetic function and they retain their own genome and replication properties. The mitochondria are also regarded as critical regulators of cell death and are the major cellular source of reactive oxygen species (ROS) (1), which cause damage to mitochondrial DNA (mtDNA) in human vascular smooth muscle cells (VSMCs) in a number of cardiovascular pathologies (2). Thus, mitochondrial dysfunction may lead to the impairment of various aspects of tissue functioning. Angiotensin II (Ang II) has been shown to participate in physiological processes, such as sodium and water homeostasis, and vascular contraction, as well as in pathophysiological processes, including hypertrophic cell growth, endothelial dysfunction, and cardiovascular and renal remodeling (3). Ang II may induce the production of mitochondrial ROS (mtROS) and several studies have suggested that this process is dependent on the full enzymatic activity of NADPH oxidases and the activation of ATP-sensitive potassium channels $\left(\mathrm{K}_{\text {ATP }}\right)(3,4)$. Furthermore, the interplay between the mitochondria and NADPH oxidase-derived $\mathrm{O}^{-2}$ constitutes a feed-forward cycle (5) and Ang II-induced ROS production may cause mitochondrial dysfunction in VSMCs (6). Since the mitochondria are both a target and source of ROS, antioxidant 
strategies specifically targeting the mitochondria may prove to be a therapeutically beneficial approach for the treatment of a number of ROS-induced cardiovascular diseases (3).

Astragaloside IV (As-IV; 3-O- $\beta$-D-xylopyranosyl-6-O$\beta$-D-glucopyranosyl cycloastragenol), is a natural saponin purified from Astragalus membranaceus (Fisch.) Bge., a traditional Chinese herb that has been widely used in clinical practice for the treatment of cardiovascular diseases (7). The herb exhibits antioxidant effects through the inhibition of ROS production, the reduction of lipid peroxidation and the stimulation of antioxidant enzymes (8). As-IV has been reported to possess diverse pharmacological properties, including antiinflammatory $(9,10)$, anti-apoptotic (11), anti-infarction (12), anti-hypertensive (13), anti-diabetic (14), anti-heart failure and myocardial protective properties $(15,16)$. Moreover, As-IV has been shown to have antioxidant properties in various types of cells, such as human umbilical endothelial (17), human mesangial (18), SK-N-SH (19) and myocardial cells (20), and plays a role in the treatment of ischemia and reperfusion injury through energy regulatory mechanisms (21). Since Ang II-induced ROS production may cause mitochondrial dysfunction in VSMCs (6), previous findings have suggested a possible role for As-IV as a therapeutic agent against mitochondrial dysfunction $(8,17-20)$. Thus, the present study was carried out to investigate the protective effects of As-IV against Ang II-induced mitochondrial injury in VSMCs and to explore the potential mechanisms responsible for these effects.

\section{Materials and methods}

As-IV preparation. As-IV was purchased from Spring \& Autumn Biological Engineering Co., Ltd., (Nanjing, China) and its purity was shown to be $>98 \%$ by high-performance liquid chromatography (HPLC) analysis and thin layer chromatography (TLC) with a single dot (data not shown). As-IV was completely dissolved in hydroxypropyl-beta-cyclodextrin (HPBCD; Sigma, St. Louis, MO, USA) to obtain a stock solution at a concentration of $6 \mathrm{mg} / \mathrm{ml}$ and subsequently diluted to $50 \mu \mathrm{g} / \mathrm{ml}$ with incubation medium to prepare a working solution. Preliminary experiments revealed that As-IV at this concentration demonstrated a more prominent pharmacodynamic effect (data not shown).

Cell culture and treatments. The VSMCs derived from the thoracic aorta were obtained from male Sprague-Dawley (SD) rats (weighing 180-200 g). The rats were purchased from the Animal Center of Nanjing Medical University (Nanjing, China). The animal experimental procedures were carried out in accordance with the Experimental Animal Ethics rules and regulations of the Ethics Committee for Animal Experimentation of Nanjing Medical University. The vascular media of the thoracic aortas were isolated from the rats under sterile conditions. The media were minced and plated into a cell culture bottle. The tissue was cultured in Dulbecco's modified Eagle's medium (DMEM), Wisent Inc., Montreal, QC, Canada) with $10 \%$ (v/v) fetal bovine serum (FBS; Gibco, Grand Island, NY, USA), $100 \mathrm{IU} / \mathrm{ml}$ penicillin and $100 \mathrm{~g} / \mathrm{ml}$ streptomycin at $37^{\circ} \mathrm{C}$ in a humidified atmosphere containing $5 \% \mathrm{CO}_{2}$. When the newly grown cells reached $80-90 \%$ confluence around the tissue, the cells were harvested by trypsin (Sigma) enzyme-digestion and were passaged in vitro for use in later experiments. VSMCs were identified by immunocytochemistry with anti- $\alpha$-smooth muscle actin antibody (1:100; ab5694, Abcam, Cambridge, UK) and by morphological anlaysis. VSMCs between passages 3 and 5 were used in the present study. When the cells reached $70-80 \%$ confluence, and they were subjected to $24 \mathrm{~h}$ of starvation in serum-free DMEM. The cells were then incubated in DMEM supplemented with Ang II (Sigma) at a concentration of $1 \mu \mathrm{M}$ and $5 \%$ FBS for $24 \mathrm{~h}$ and subsequently treated with either a mixture of Ang II $(1 \mu \mathrm{M})$ and As-IV $(50 \mu \mathrm{g} / \mathrm{ml})$ or Ang II $(1 \mu \mathrm{M})$ alone for a further $24 \mathrm{~h}$. The untreated controls were maintained in DMEM only with 5\% FBS for $48 \mathrm{~h}$. In addition, this study protocol was approved by the Medical Ethics Committee of the First Affiliated Hospital of Nanjing Medical University, Nanjing, China.

Transmission electron microscopy (TEM). Following treatment, the VSMCs were collected and fixed in $2.5 \%$ glutaraldehyde overnight, and this was followed by secondary fixation in $1 \%$ osmium tetroxide for $1 \mathrm{~h}$. The fixed specimens were then dehydrated through a graded series of ethanol to $100 \%$ and embedded in TAAB Epon (Marivac Canada Inc., St. Laurent, QC, Canada). Ultrathin sections $(60 \mathrm{~nm})$ were cut, placed onto copper grids, stained with uranyl acetate and lead citrate prior to examination using a transmission electron microscope (Tecnai G2 Spirit BioTWIN; FEI, Hillsboro, OR, USA) at an accelerating voltage of $80 \mathrm{kV}$.

Measurement of mitochondrial bioenergetic function by extracellular flux (XF) analysis. The XF96 Extracellular Flux Analyzer (Seahorse Bioscience, Billerica, MA, USA) was used to measure the rate changes in extracellular flux of dissolved $\mathrm{O}_{2}$ and protons in the medium immediately surrounding the adherent intact cells cultured on a XF96-well microplate (Seahorse Bioscience). The VSMCs were seeded in XF96-well microplates at $8.0 \times 10^{3}$ cells/well $\left(0.32 \mathrm{~cm}^{2}\right)$ in $80 \mu \mathrm{l}$ DMEM medium with $10 \% \mathrm{FBS}$ and incubated at $37^{\circ} \mathrm{C}$ with $5 \% \mathrm{CO}_{2}$ for $24 \mathrm{~h}$. The VSMCs were then treated as described above. The medium was removed and replaced with assay medium $1 \mathrm{~h}$ prior to the beginning of the assay and maintained at $37^{\circ} \mathrm{C}$. After taking baseline measurements of the mitochondrial oxygen consumption rates (OCRs), the OCRs were measured using the XF Cell Mito Stress Test kit (Seahorse Bioscience), and the following were sequentially added to each well: oligomycin (1 $\mu \mathrm{M}$, blocker of the mitochondrial complex $\mathrm{V}$, inhibiting the electron chain from being coupled to ATP synthesis), FCCP ( $2 \mu \mathrm{M}$, an uncoupling agent which allows maximum electron transport) and rotenone ( $1 \mu \mathrm{M}$, mitochondrial complex I blocker which eliminates mitochondrial respiration), as prevoiusly described (22). A typical OCR trace on how each parameter is derived is shown in Fig. 1B (panel a). Values were normalized to the cell number $\left(10^{5}\right.$ cells as one unit) per well by cell counting on completion of the XF assay.

Measurement of mitochondrial ATP production. Following treatment, the VSMCs were collected and disrupted in $200 \mu \mathrm{l}$ of ice-cold lysis buffer from the luciferase-based luminescence ATP detection kit (Beyotime Institute of Biotechnlogy, Nantong, China). Following centrifugation at $12,000 \times \mathrm{g}$ for $5 \mathrm{~min}$ at $4^{\circ} \mathrm{C}$, ATP detection working solution $(100 \mu \mathrm{l})$ was added to each well of a black 96-well culture plate which was then incubated for 
$3 \mathrm{~min}$ at room temperature. Cell lysate $(40 \mu \mathrm{l})$ was then added to the wells of the culture plate, and luminescence was measured immediately using a luminometer (Turner BioSystems, Sunnyvale, CA, USA). A standard curve was generated from the protein concentration of each well using the BCA Protein assay kit (Thermo Fisher Scientific, Waltham, MA, USA). The ATP level in each sample was expressed as nmol/mg protein.

Measurement of ROS production. Following treatment, the VSMCs were incubated with MitoSOX ${ }^{\mathrm{TM}}$ Red mitochondrial superoxide indicator (Molecular Probes, Inc., Eugene, OR, USA) at $37^{\circ} \mathrm{C}$ for $20 \mathrm{~min}$ and washed gently 3 times with warm buffer, and then imaged using a confocal microscope (original magnification, x200). Mitochondrial ROS generation was expressed as the mean fluorescence intensity of the red color, as previously described (23).

Measurement of mitochondrial membrane potential $(\Delta \Psi m)$. Following treatment, the VSMCs were stained with $2 \mu \mathrm{mol} / 1$ of JC-1 (Beyotime Institute of Biotechnology) probe for 30 min and rinsed twice with PBS and then imaged using a confocal microscope (original magnification, $\mathrm{x} 400) . \Delta \Psi_{\mathrm{m}}$ was determined by monitoring the dual emissions from the mitochondrial JC-1 monomers (green) and the aggregates (red) under a confocal microscope under $488 \mathrm{~nm}$ laser excitation. $\Delta \Psi \mathrm{m}$ was expressed as the emission intensity ratio (the ratio of green to red fluorescence) which represents the relative arbitrary $\Delta \Psi \mathrm{m}$ level. A higher ratio indicates a lower $\Delta \Psi \mathrm{m}$.

Measurement of ROS-related enzyme activity. Following treatment, the total cellular protein from the VSMCs was prepared as previously described (24), and the protein concentration was assessed using a BCA protein assay kit (Thermo Fisher Scientific). The activities of NADPH oxidase, xanthine oxidase and manganese superoxide dismutase (Mn-SOD) were measured using an NADPH oxidase activity quantitative assay kit (Genmed Scientifics Inc., Wilmington, DE, USA), a xanthine oxidase assay kit (Nanjing Jiancheng Bioengineering Institute, Nanjing, China) and a $\mathrm{CuZn/Mn-SOD}$ assay kit (Beyotime Institute of Biotechnology) according to the manufacturer's instructions, respectively. The values were calculated according to the manufacturer's instructions and standardized to the protein concentration of the cells.

Reverse transcription-quantitative polymerase chain reaction $(R T-q P C R)$. Following treatment, total RNA was purified from the VSMCs using TRIzol reagent (Invitrogen, Carlsbad, CA, USA) according to the manufacturer's instructions. Total RNA $(1 \mu \mathrm{g})$ was reverse transcribed into cDNA using the PrimeScriptTM RT reagent kit (Takara Bio, Dalian, China), and then the cDNA was amplified with SYBR Premix Ex Taq ${ }^{\mathrm{TM}}$ (Taka a Bio) under the following cycling conditions: $30 \mathrm{sec}$ at $95^{\circ} \mathrm{C}$ for 1 cycle, followed by 40 cycles of $5 \mathrm{sec}$ at $95^{\circ} \mathrm{C}$ and $30 \mathrm{sec}$ at $60^{\circ} \mathrm{C}$. The primer sequences were as follows: peroxisome proliferator-activated receptor-gamma coactivator-1 $\alpha$ (PGC-1 $\alpha$ ) sense, 5'-CGG AGC AAT CTG AGT TAT ACG-3' and antisense, 5'-CAG TCA CAG GAG GCA TCT-3'; mitochondrial transcription factor A (Tfam) sense, 5'-ATC TCA TCC GTC GCA GTG-3' and antisense, 5'-GCA CAG TCT TGA TTC CAG TTC-3'; and GAPDH sense, 5'-GTG AAG GTC GGT GTG AAC-3' and antisense, 5'-GGT GAA GAC GCC AGT AGA-3'. The threshold cycle $(\mathrm{Ct})$ value was set within the exponential phase of the PCR. The relative quantities of the different mRNAs were calculated by comparing the cycle times for each target PCR. The target PCR Ct values were normalized by subtracting the $\mathrm{Ct}$ (GAPDH) value, and then the relative mRNA expression was calculated

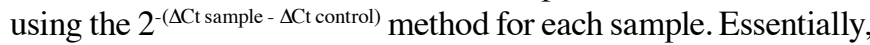
the same protocol was used for mtDNA quantification, normalizing the Rnr2 gene amplification level against the GAPDH gene. The primer sequences were as follows: Rnr2 sense, 5'-AGC TAT TAA TGG TTC GTT TGT-3' and antisense, 5'-AGG AGG CTC CAT TTC TCT TGT-3'; and GAPDH sense, 5'-GGA CCT CAT GGC CTA CAT GG-3' and antisense, 5'-ATT CGA GAG AAG GGA GGG CT-3'. The ratio of mtDNA (Rnr2) to nDNA (GAPDH) was used as an estimate of the number of mitochondria per cell.

Western blot analysis. Total cellular protein from the treated VSMCs was extracted following standard protocols (24) and the protein concentration of the supernatant was determined using the BCA protein assay kit (Thermo Fisher Scientific). Equal amounts of protein $(30 \mu \mathrm{g})$ were electrophoretically separated by $8 \%$ SDS-PAGE and then transferred onto polyvinylidene difluoride membranes. After blocking (non-fat milk 5\%, 2 h), the membranes were incubated at $4^{\circ} \mathrm{C}$ overnight with the following primary antibodies: anti-PGC-1 $\alpha$ antibody $(1: 1,000$; ab54481; Abcam), anti-dynamin 1-like protein 1 (Drp1) antibody (1:1,000; ab56788, Abcam), anti-parkin antibody (1:1,000; Abcam, ab179812) and anti-GAPDH antibody (1:1,000; 5174P; Cell Signaling Technology, Inc., Danvers, MA, USA). After washing, the membranes were incubated with HRP-conjugated secondary antibodies, including anti-rabbit antibody (1:5,000; ab6721, Abcam) and anti-mouse antibody (1:2,000; ab97023, Abcam) at room temperature. Signals were detected with SuperSignal West Pico chemiluminescent substrate (Thermo Fisher Scientific), and the intensity of the protein band was quantified using Gel-Pro Analyzer software. Intensity values were normalized to GAPDH and expressed as the relative protein expression.

Statistical analysis. Data are expressed as the means \pm SEM of a certain number of independent experiments (the exact number of experiments performed is indicated in each figure legend). Statistical analysis was performed by a Student's t-test or by one-way ANOVA followed by post hoc tests. A value of $\mathrm{P}<0.05$ was considered to indicate a statisticallydifference.

\section{Results}

Damaging effects of Ang II on the morphology and function of mitochondria in VSMCs. To confirm the effects of As-IV treatment, we evaluated the extent of Ang II-induced mitochondrial damage by assessing mitochondrial morphology and bioenergetic function. Our results revealed that the mitochondria in the VMSCs treated with Ang II $(1 \mu \mathrm{M})$ for $24 \mathrm{~h}$ were swollen, and vacuolization had occurred with almost a complete loss of cristae (Fig. 1A). In addition, treatment with Ang II $(1 \mu \mathrm{M})$ for $24 \mathrm{~h}$ markedly reduced the basal OCR, ATP-linked OCR, maximal OCR and spare respiratory capacity by 19.6, 16.8, 24.1 and $30.5 \%$ compared with the untreated VSMCs, respec- 

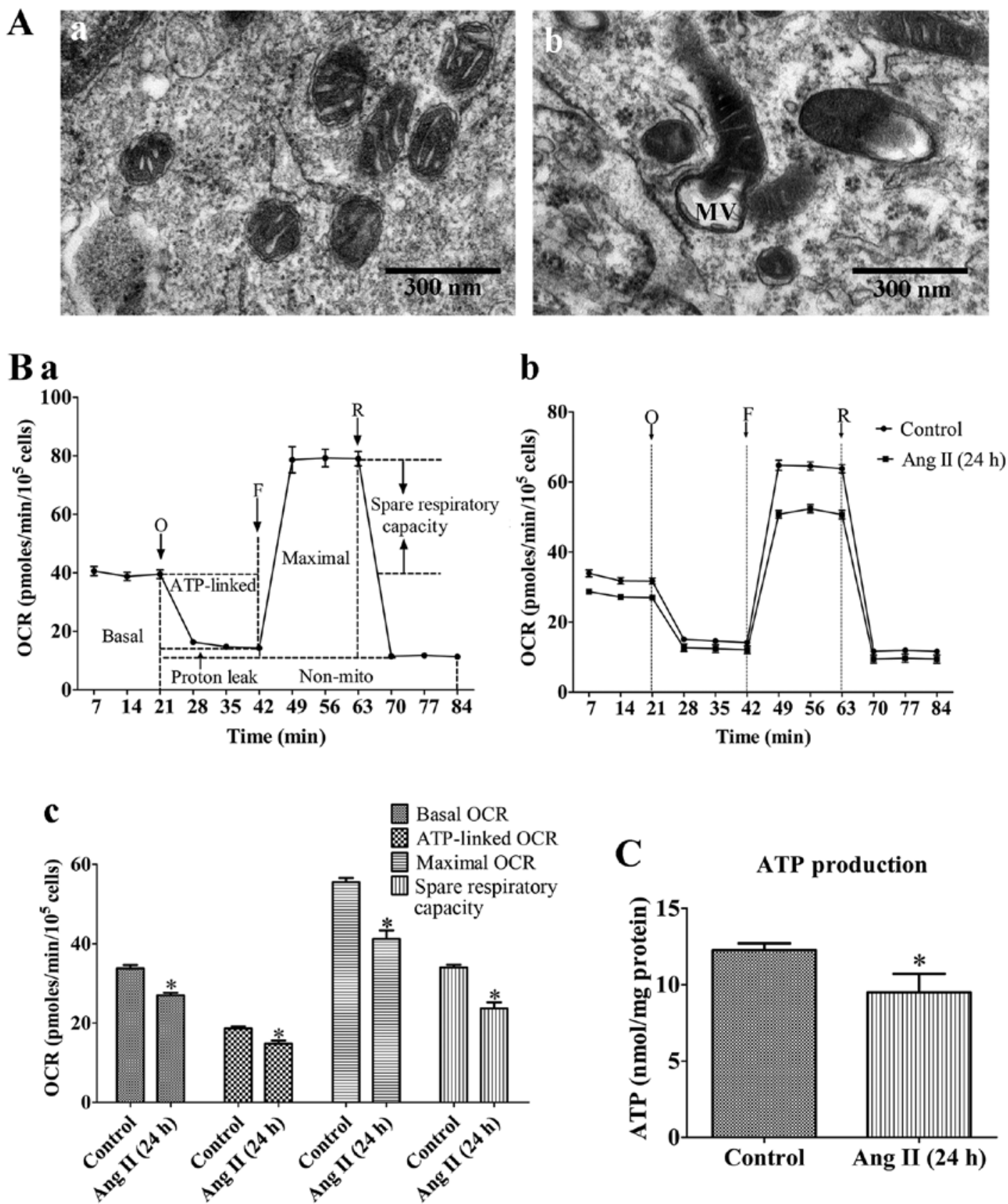

Figure 1. Mitochondrial dysfunction of rat VSMCs induced by angiotensin II (Ang II; $1 \mu \mathrm{M}$ ) treatment for $24 \mathrm{~h}$. (A) Transmission electron micrographs of mitochondrial morphology in VSMCs (original magnification, x80,000). (Panel a) Untreated VSMCs; (panel b) VSMCs treated with Ang II (1 $\mu \mathrm{M})$ for $24 \mathrm{~h}$. The mitochondria in the VMSCs treated with Ang II $(1 \mu \mathrm{M})$ for $24 \mathrm{~h}$ were swollen, and vacuolization had occurred with almost a complete loss of cristae. MV, mitochondrial vacuolization; scale bar, $300 \mathrm{~nm}$. (B) Assessment of mitochondrial function in VSMCs. (Panel a) Schematic representation of the mitochondrial function assay, including basal oxygen consumption rate (OCR), ATP-linked OCR, maximal OCR, spare respiratory capacity, proton leak and non-mito OCR (25). O, oligomycin; F, FCCP; R, rotenone. (Panel b) Real-time analysis of mitochondrial OCRs. (Panel c) Quantitative analysis of the effect of Ang II on mitochondrial OCR readings. Results are the means \pm SEM of 3 independent experiments (data were averaged from 12-16 duplicate wells). (C) Effects of Ang II on cellular ATP production. Control, untreated VSMCs; Ang II ( $24 \mathrm{~h})$, VSMCs treated with Ang II $(1 \mu \mathrm{M})$ for $24 \mathrm{~h}$. Data represents the means \pm SEM of 5 independent experiments. "P $<0.05$ vs. untreated VSMCs.

tively (Fig. 1B). Furthermore, treatment with Ang II $(1 \mu \mathrm{M})$ for $24 \mathrm{~h}$ reduced cellular ATP production compared with the untreated cells (Fig. 1C). These results demonstrated that the VSMCs treated with Ang II $(1 \mu \mathrm{M})$ for $24 \mathrm{~h}$ exhibited signs of damage; abnormal mitochondrial morphology and impaired bioenergetic function.

Beneficial effects of As-IV on damaged mitochondria in VSMCs. To determine the beneficial effects of As-IV on damaged mitochondria, we used TEM to observe the mitochondrial ultramicrostructure. TEM revealed that the mitochondrial morphology remained intact in the untreated VSMCs, whereas the mitochondria from the VMSCs treated with Ang II $(1 \mu \mathrm{M})$ for $48 \mathrm{~h}$ were swollen and vacuolization had occurred with almost a complete loss of cristae. Furthermore, some of the mitochondria contained electron dense deposits in their matrices and exhibited whorl-like inner membrane defects. By contrast, the mitochondria from the VSMCs treated 

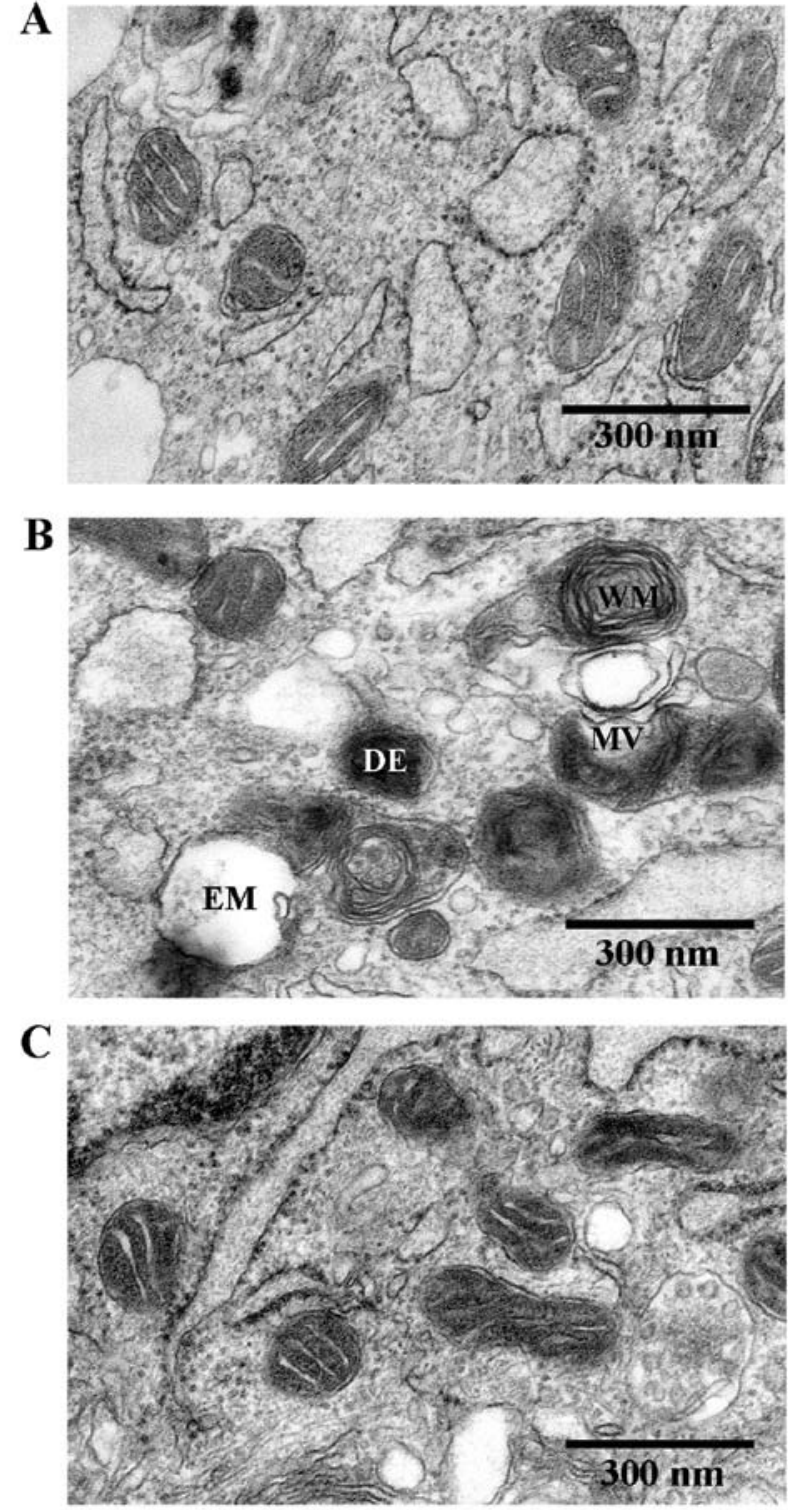

Figure 2. Transmission electron micrographs of mitochondrial morphology in VSMCs (original magnification, x80,000). (A) Untreated VSMCs; mitochondrial morphology remained intact in the untreated VSMCs. (B) VSMCs treated with angiotensin II (Ang II; $1 \mu \mathrm{M}$ ) for $48 \mathrm{~h}$; mitochondria were swollen and vacuolization had occurred with almost a complete loss of cristae. Some of the mitochondria contained electron dense deposits in their matrices and exhibited whorl-like inner membrane defects. (C) VSMCs treated with astragaloside IV (As-IV; $50 \mu \mathrm{g} / \mathrm{ml}$ ) for a further $24 \mathrm{~h}$ following exposure to Ang II for $24 \mathrm{~h}$ exhibited a basically normal morphology and no significant ultrastructural damage. EM, empty mitochondria; MV, mitochondrial vacuolization; WM, whorl-like inner membrane; DE, dense electron. Scale bar, $300 \mathrm{~nm}$.

with As-IV (50 $\mu \mathrm{g} / \mathrm{ml})$ for $24 \mathrm{~h}$ following exposure to Ang II for $24 \mathrm{~h}$, exhibited a basically normal morphology and no significant ultrastructural damage (Fig. 2).

Effects of As-IV on mitochondrial bioenergetic function. To demonstrate the bioenergetic function of the mitochondria in VSMCs following treatment with Ang II and the effects of As-IV on mitochondrial energy metabolism, we exposed the VSMCs to Ang II for $24 \mathrm{~h}$, and then added As-IV $(50 \mu \mathrm{g} / \mathrm{ml})$ for a further $24 \mathrm{~h}$. Treatment with Ang II $(1 \mu \mathrm{M})$ for $48 \mathrm{~h}$ mark-
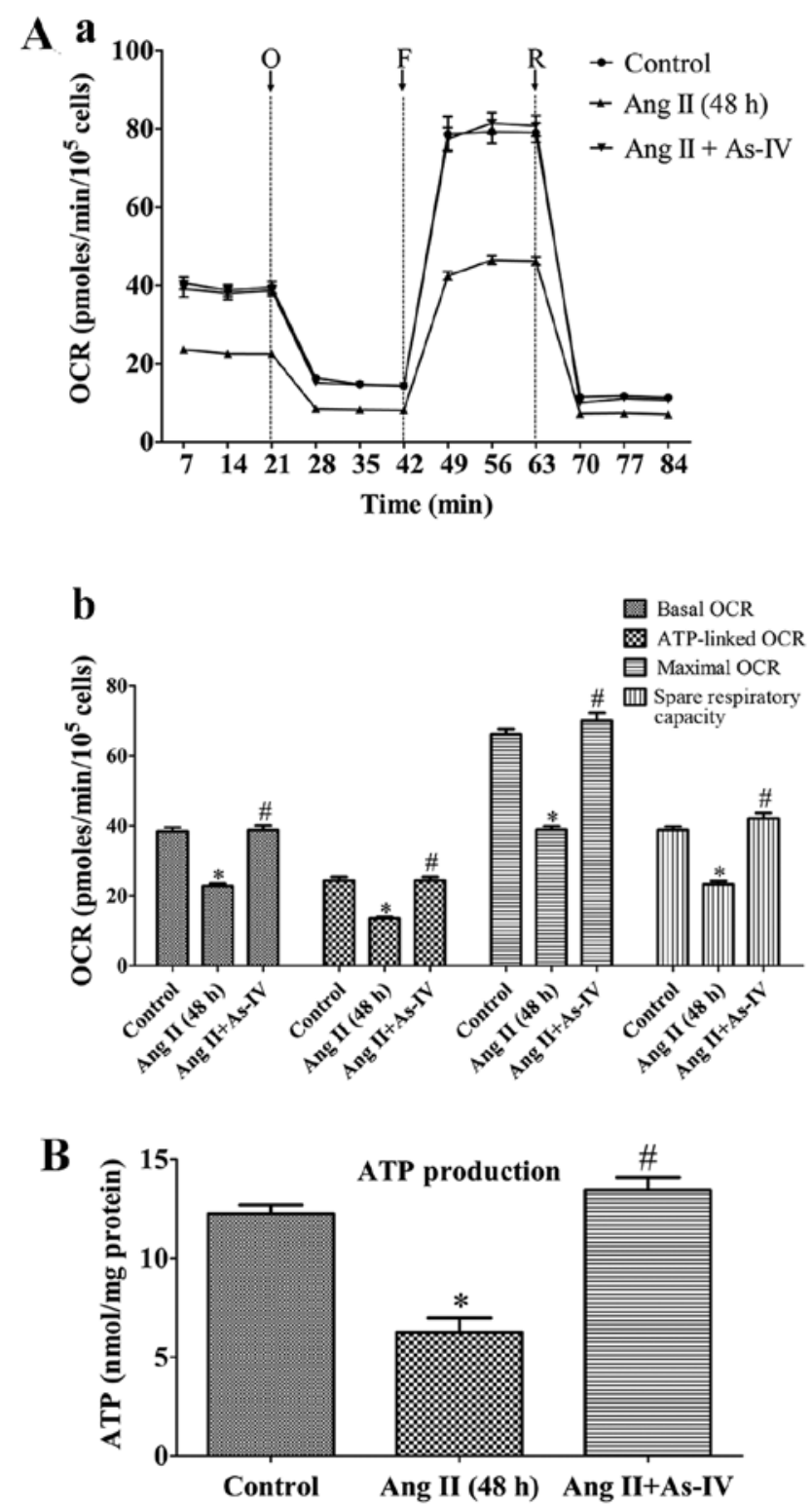

Figure 3. Effects of astragaloside IV (As-IV) on mitochondrial bioenergetic function. (A) Assessment of mitochondrial function in VSMCs. (Panel a) Real-time analysis of mitochondrial OCRs. O, oligomycin; F, FCCP; R, rotenone. (Panel b) Quantitative analysis of the effects of As-IV on mitochondrial OCRs. Results are the means \pm SEM of 3 independent experiments (data were averaged from 12-16 duplicate wells). (B) Effects of As-IV on angiotensin II (Ang II)-induced reduction of cellular ATP production. Control, untreated VSMCs; Ang II (48 h), VSMCs treated with Ang II (1 $\mu \mathrm{M})$ for $48 \mathrm{~h}$; Ang II + As-IV, VSMCs treated with As-IV $(50 \mu \mathrm{g} / \mathrm{ml})$ for a further $24 \mathrm{~h}$ following exposure to Ang II for $24 \mathrm{~h}$. Data represent the means \pm SEM of 5 independent experiments. ${ }^{*} \mathrm{P}<0.05$ vs. untreated VSMCs; ${ }^{\#} \mathrm{P}<0.05$ vs. VSMCs treated with Ang II $(1 \mu \mathrm{M})$ for $48 \mathrm{~h}$.

edly reduced the basal OCR, ATP-linked OCR, maximal OCR and spare respiratory capacity by 40.6, 44.4, 41.2 and $39.9 \%$ compared with the untreated VSMCs, respectively. By contrast, treatment with As-IV $(50 \mu \mathrm{g} / \mathrm{ml})$ significantly increased the mitochondrial respiratory functions in the VSMCs, so that there was almost no difference with the untreated VSMCs (Fig. 3A). Treatment with Ang II $(1 \mu \mathrm{M})$ for $48 \mathrm{~h}$ also reduced cellular ATP production compared with the controls, whereas treatment with As-IV $(50 \mu \mathrm{g} / \mathrm{ml})$ reversed the decrease in ATP production (Fig. 3B). 


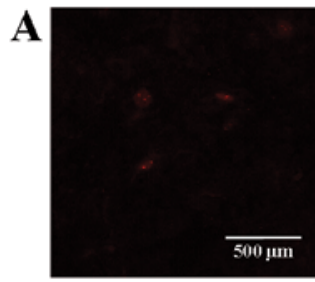

$\boldsymbol{2}$

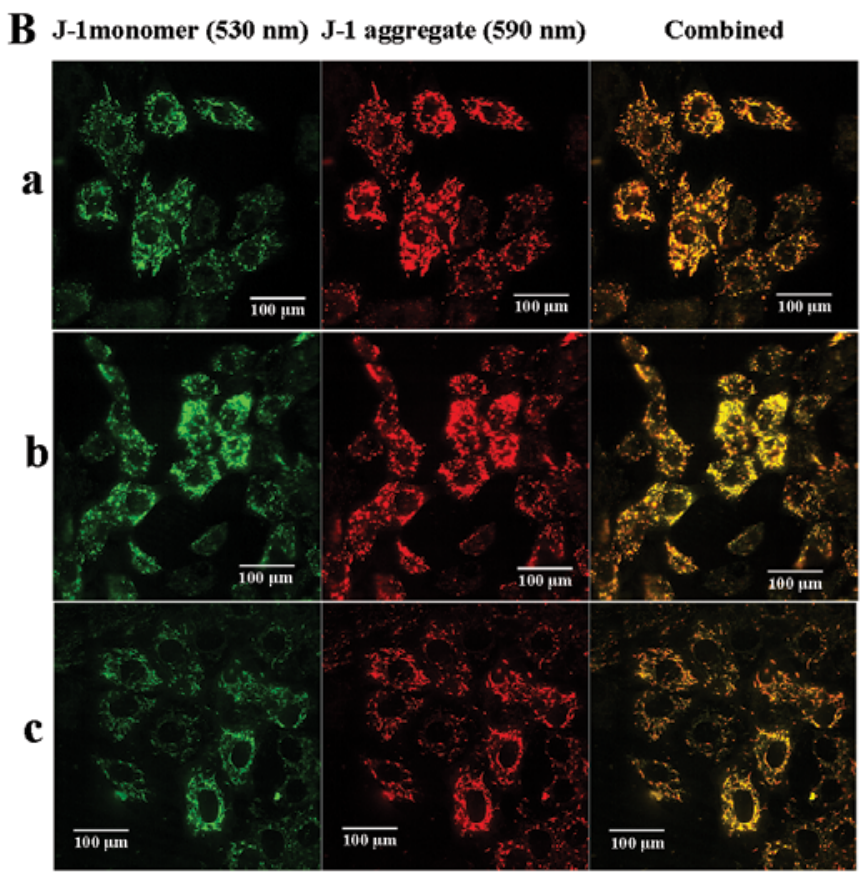

C

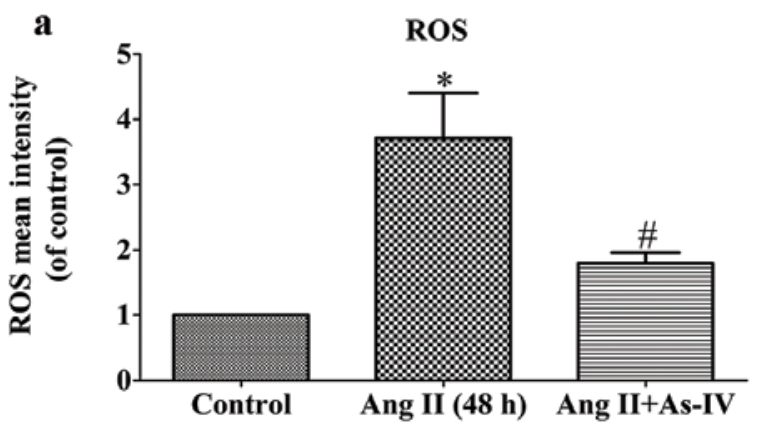

b

$\Delta \Psi \mathrm{m}$

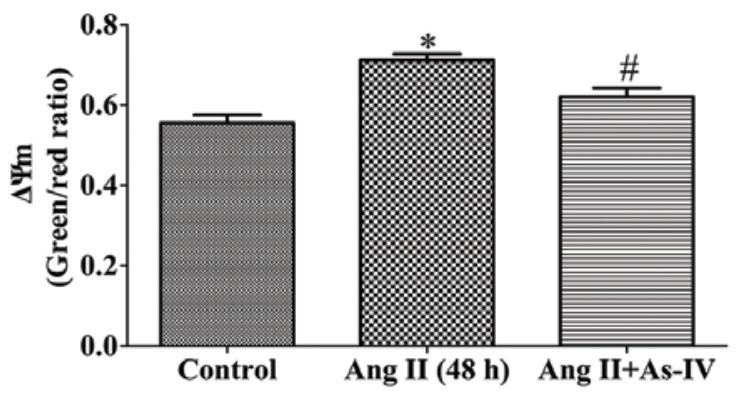

Figure 4. Effects of As-IV on excessive generation of mitochondrial ROS (mtROS) and mitochondrial membrane potential ( $\Delta \Psi \mathrm{m})$. (A) Imaging of mtROS using confocal microscopy (original magnification, $\mathrm{x} 200$ ). Scale bar, $500 \mu \mathrm{m}$. (B) Imaging of $\Delta \Psi \mathrm{m}$ using confocal microscopy (original magnification, $\mathrm{x} 400$ ). Orange color indicates the combination of green (JC-1 monomer) and red (JC-1 aggregate). Scale bar, $100 \mu \mathrm{m}$. (Panel a) Untreated VSMCs; (panel b) VSMCs treated with angiotensin II (Ang II; $1 \mu \mathrm{M}$ ) for $48 \mathrm{~h}$; (panel c) VSMCs treated with astragaloside IV (As-IV; $50 \mu \mathrm{g} / \mathrm{ml}$ ) for a further $24 \mathrm{~h}$ following exposure to Ang II for $24 \mathrm{~h}$. (C) Quantitative analysis of the effects of As-IV on mtROS generation (panel a) and $\Delta \Psi \mathrm{m}$ (panel b). Control, untreated VSMCs; Ang II (48 h), VSMCs treated with Ang II $(1 \mu \mathrm{M})$ for $48 \mathrm{~h}$; Ang II + As-IV, VSMCs treated with As-IV $(50 \mu \mathrm{g} / \mathrm{ml})$ for a further $24 \mathrm{~h}$ following exposure to Ang II for $24 \mathrm{~h}$. Data represent the means \pm SEM of 5 independent experiments. ${ }^{*} \mathrm{P}<0.05$ vs. untreated VSMCs; ${ }^{~} \mathrm{P}<0.05$ vs. VSMCs treated with Ang II $(1 \mu \mathrm{M})$ for $48 \mathrm{~h}$.

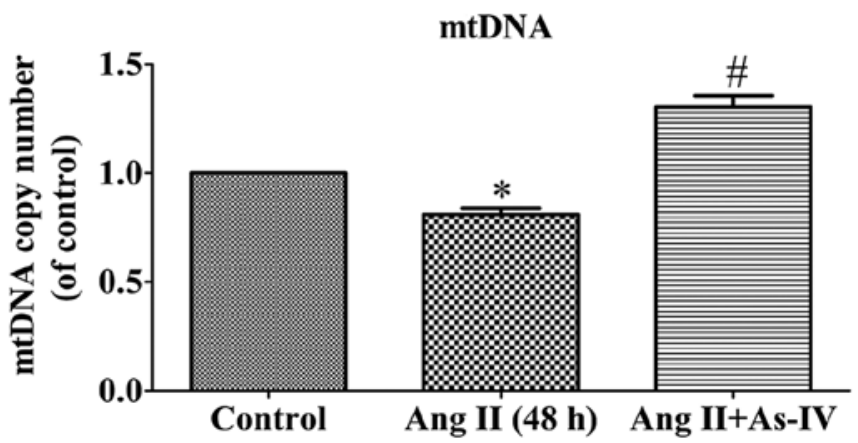

Figure 5. Effects of astragaloside IV (As-IV) on cellular mtDNA content variation caused by angiotensin II (Ang II). Control, untreated VSMCs; Ang II (48 h), VSMCs treated with Ang II $(1 \mu \mathrm{M})$ for $48 \mathrm{~h}$; Ang II + As-IV, VSMCs treated with As-IV $(50 \mu \mathrm{g} / \mathrm{ml})$ for a further $24 \mathrm{~h}$ following exposure to Ang II for $24 \mathrm{~h}$. Data represent the means \pm SEM of 5 independent experiments. ${ }^{*} \mathrm{P}<0.05$ vs. untreated VSMCs; ${ }^{\prime} \mathrm{P}<0.05$ vs. VSMCs treated with Ang II $(1 \mu \mathrm{M})$ for $48 \mathrm{~h}$.

As-IV prevents the Ang II-induced excessive generation of $m t R O S$ and the decrease in $\Delta \Psi m$. Ang II-induced mtROS production may be a potential mechanism responsible for mitochondrial dysfunction (3) and the Ang II-induced depolarization of $\Delta \Psi \mathrm{m}$ has been shown to be dependent on ROS (6). In this study, compared with the untreated VSMCs, a significant increase in the generation of mtROS and a decrease in $\Delta \Psi \mathrm{m}$ (shown by an increase in the green/red fluorescence ratio) was observed in the Ang II-treated VSMCs. However, treatment of the VSMCs with As-IV reversed these effects, with no significant changes observed in mtROS generation and $\Delta \Psi \mathrm{m}$ compared to the controls (Fig. 4).

As-IV inhibits the Ang II-induced decrease in mtDNA copy numbers in VSMCs. mtDNA is particularly susceptible to oxidative damage resulting from ROS production in the mitochondrial matrix and the lack of DNA protective histones. Maintaining an adequate copy number of mtDNA is related to the energy demands that sustain normal function and it is crucial for cell viability (26). As shown by our results, the mtDNA copy number of the Ang II-treated VSMCs was lower than that of the untreated VSMCs. Compared with Ang II treatment alone, the addition of As-IV to the medium significantly increased the mtDNA copy number (Fig. 5). 

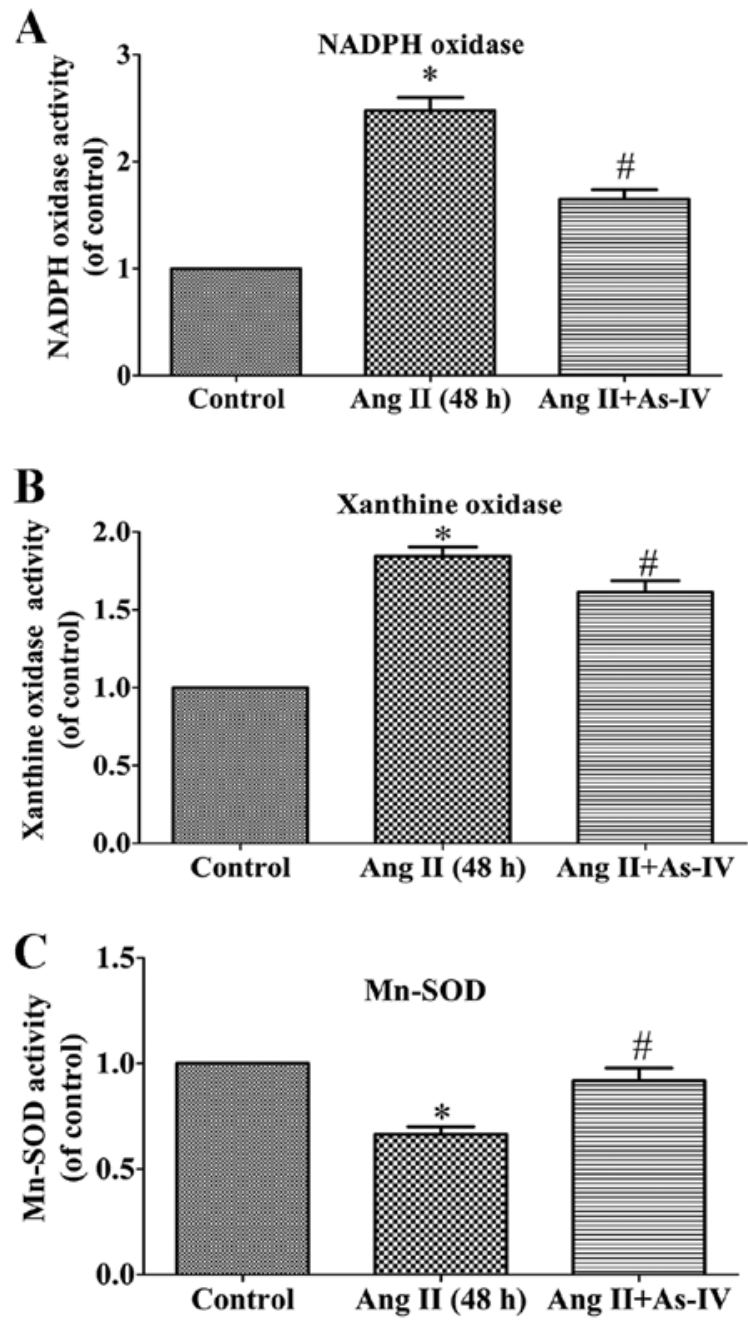

Figure 6. Effects of astragaloside IV (As-IV) on enzyme activity associated with ROS generation and scavenging. As-IV significantly attenuated the angiotensin II (Ang II)-induced increase in NADPH oxidase activity (A) and xanthine oxidase activity (B), and stimulated Mn-SOD activity (C) in VSMCs. Control, untreated VSMCs; Ang II (48 h), VSMCs treated with Ang II (1 $\mu \mathrm{M})$ for $48 \mathrm{~h}$; Ang II + As-IV, VSMCs treated with As-IV $(50 \mu \mathrm{g} / \mathrm{ml})$ for a further $24 \mathrm{~h}$ following exposure to Ang II for $24 \mathrm{~h}$. Data represent the means \pm SEM of 5 independent experiments. ${ }^{*} \mathrm{P}<0.05$ vs. untreated VSMCs; ${ }^{*} \mathrm{P}<0.05$ vs. VSMCs treated with Ang II $(1 \mu \mathrm{M})$ for $48 \mathrm{~h}$.

As-IV attenuates the activities of NADPH oxidase and xanthine oxidase and enhances the activity of Mn-SOD. ROS are generated from different sources, including NADPH oxidases and xanthine oxidase, while $\mathrm{Mn}-\mathrm{SOD}\left(\mathrm{SOD}_{2}\right)$, which is located in the mitochondrial matrix, is an important antioxidant which regulates ROS production (27). The present study demonstrated that compared with the untreated VSMCs, the activity of NADPH oxidase (Fig. 6A) and xanthine oxidase (Fig. 6B) increased significantly in the Ang II treated VSMCs, whereas this increase in enzyme activity was alleviated by treatment with As-IV. Furthermore, As-IV also exhibited the ability to reverse the decrease in Mn-SOD activity induced by Ang II (Fig. 6C).

Effects of As-IV on the expression of genes related to mitochondrial autophagy and biogenesis. Mitochondrial autophagy is mediated by parkin and Drp1 (43), while mitochondrial biogenesis is regulated by PGC-1 $\alpha$ and Tfam (47).
In the present study, our results revealed that the protein levels of Drp1 and parkin, which are vital to mitochondrial autophagy, increased in the Ang II-treated VSMCs, and treatment with As-IV further increased the protein expression of parkin and Drp1 (Fig. 7A). On the other hand, compared with the Ang II-treated VSMCs, treatment with As-IV significantly enhanced the mRNA and protein expression of PGC-1 $\alpha$ and the mRNA expression of Tfam. By contrast, we were unable to detect significant differences in the expression of PGC- $1 \alpha$ and Tfam between the untreated VSMCs and the Ang II-treated VSMCs (Fig. 7B and C).

\section{Discussion}

The present study demonstrated that As-IV reverses the Ang II-induced mitochondrial dysfunction in VSMCs by exerting antioxidant effects against excessive ROS generation and enhancing mitochondrial autophagy and biogenesis.

In the present study, we demonstrated that Ang II, which was used at a concentration of $1 \mu \mathrm{M}$ for $24 \mathrm{~h}$, induced mitochondrial dysfunction in VSMCs (Fig. 1). These results are in accordance with those of previous studies $(6,28,29)$. Thus, VSMCs treated with Ang II $(1 \mu \mathrm{M})$ for $24 \mathrm{~h}$ were used as a cell model of mitochondrial injury prior to treatment with As-IV.

In the present study, the Ang II-treated VSMCs exhibited mitochondrial morphological abnormalities and mitochondrial bioenergetic dysfunction, including a reduction in mitochondrial OCRs and ATP production (Figs. 2 and 3). The Ang II-treated VSMCs also exhibited a decrease in $\Delta \Psi \mathrm{m}$ (Fig. 4B and C) and mtDNA damage (Fig. 5), which are typical manifestations of mitochondrial damage. Furthermore, mtROS generation was significantly increased in the Ang II-treated VSMCs (Fig. 4A and $\mathrm{C}$ ) exhibiting mitochondrial damage.

Under normal physiological conditions, the levels of ROS are generally low and they are controlled by antioxidants, such as Mn-SOD. However, under pathological conditions, it has been demonstrated that mtROS generation is triggered by elevated ROS levels, caused by the Ang II-induced activation of NADPH oxidase via AT1R-PKC signaling (30) and the downregulation of antioxidant expression, resulting in decreased scavenging capacity (31); and mtROS also plays a critical role in depressing mitochondrial energy metabolism $(32,33)$. Thus, increased mtROS generation primarily led to mitochondrial injury in the present study. Our experiment to visualize mtROS provided evidence that As-IV diminishes the Ang II-induced excessive generation of mtROS (Fig. 4A and C). These results demonstrate that As-IV exerts its antioxidant effects against mitochondrial injury through the attenuation of the excessive generation of mtROS.

The physiological generation of ROS occurs through several mechanisms, including leakage from the electron transport chain and as a metabolic byproduct of enzymes, such as xanthine oxidase (34). Ang II-induced NADPH oxidase activation is an example of a system that generates ROS not as a byproduct, but rather as the primary function of the enzyme system (35). On the other hand, there is growing evidence to suggest that the Ang II-induced activation of xanthine oxidase may be secondary to ROS generation from other sources, through the thiol oxidation of the xanthine dehydrogenase sulfhydryl residues which contribute, to a certain extent, to 

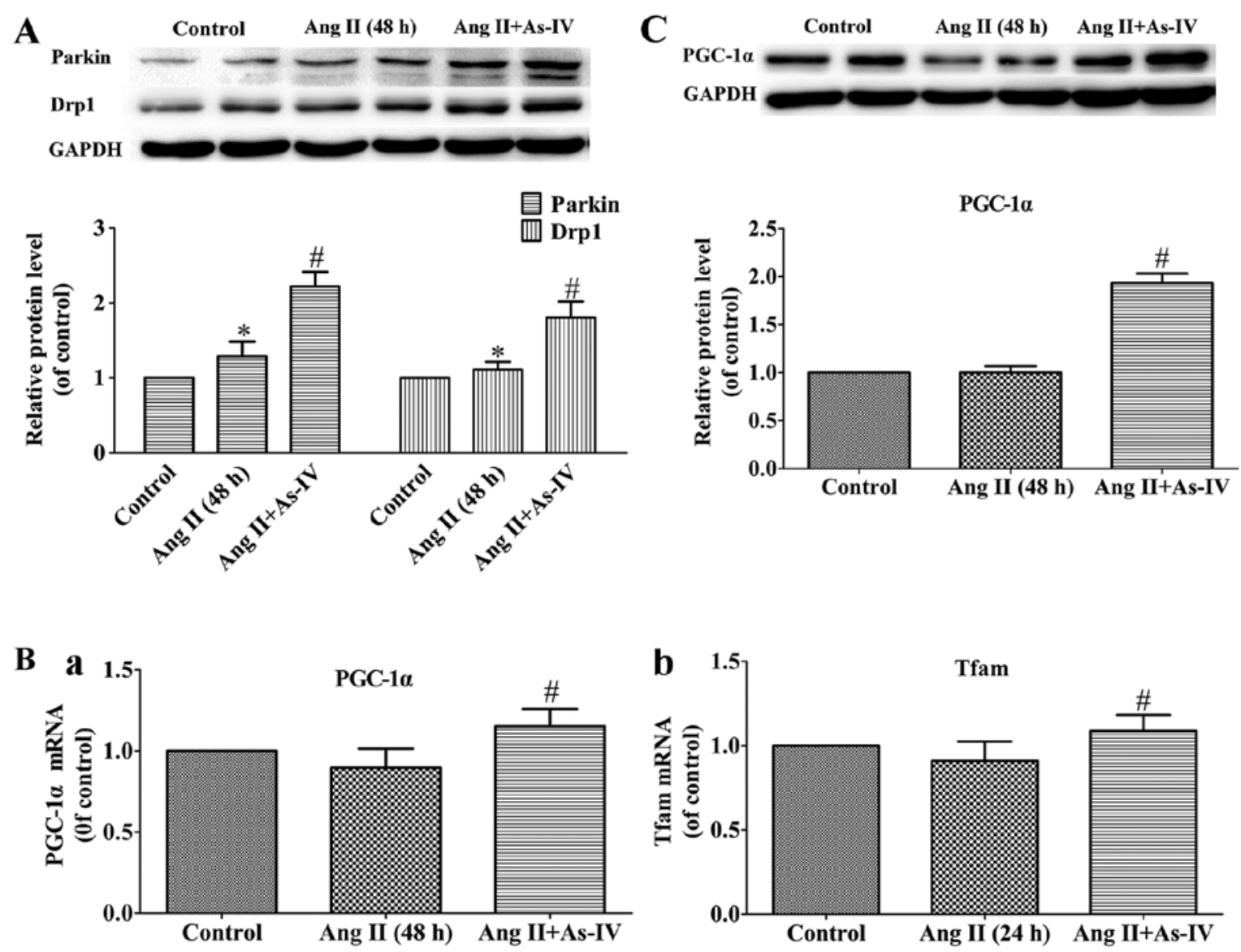

Figure 7. Effects of astragaloside IV (As-IV) on variations of mRNA and protein expression. (A) Protein expression of parkin and dynamin 1-like protein 1 (Drp1). (B) mRNA expression of (panel a) peroxisome proliferator-activated receptor-gamma coactivator-1 $\alpha$ PGC-1 $\alpha$ and (panel b) mitochondrial transcription factor A (Tfam). (C) Protein expression of PGC-1 $\alpha$. Control, untreated VSMCs; Ang II (48 h), VSMCs treated with angiotensin II (Ang II; $1 \mu \mathrm{M})$ for 48 h; Ang $\mathrm{II}+$ As-IV, VSMCs treated with As-IV $(50 \mu \mathrm{g} / \mathrm{ml})$ for a further $24 \mathrm{~h}$ following exposure to Ang II for $24 \mathrm{~h}$. Each Data represent the means \pm SEM (n=5). ${ }^{*} \mathrm{P}<0.05$ vs. untreated VSMCs; ${ }^{\#} \mathrm{P}<0.05$ vs. VSMCs treated with Ang II $(1 \mu \mathrm{M})$ for $48 \mathrm{~h}$.

Ang II-induced ROS generation $(34,36)$. In this study, we found that Ang II induced an increase in NADPH oxidase and xanthine oxidase activity, and this effect was significantly inhibited by treatment with As-IV (Fig. 6A and B).

In general, the maintenance of ROS production is normally counteracted and an equilibrium oxidative state is retained by SODs, which catalyze the dismutation of $\mathrm{O}^{-2}$ into oxygen and $\mathrm{H}_{2} \mathrm{O}_{2}$, thereby serving a key antioxidant function (37). It has been suggested that $\mathrm{Mn}-\mathrm{SOD}\left(\mathrm{SOD}_{2}\right)$, one of the three isoforms of SOD, is one of the important defenses against oxidative damage within the mitochondria (27). The beneficial effects of As-IV on Mn-SOD activity in renal proximal tubular cells (38) and $\mathrm{H} 9 \mathrm{c} 2$ cells (39) have been demonstrated. Our experiment on the activity of Mn-SOD revealed that As-IV possessed the ability to reverse the reduction in mitochondrial Mn-SOD activity indcued by Ang II (Fig. 6C). Taken together with the findings that As-IV attenuates the activity of NADPH oxidase and xanthine oxidase, these results suggest that As-IV reverses the Ang II-induced excessive production of mtROS by inhibiting $m t R O S$ generation and stimulating the elimination of $m$ tROS.

In our experiment on the visualization of mitochondrial morphology, we found that the VSMCs treated with Ang II exhibited abnormal mitochondrial ultrastructures, while the morphology of the mitochondria from VSMCs treated with
As-IV was basically normal. Previous studies have suggested that mitochondria damaged by ROS need to be selectively removed to lysosomes and degraded in a process known as mitophagy, to protect the cells from cell death $(40,41)$. They may then be substituted by the progeny of more bioenergetically active mitochondria (42). Thus, we speculated that treatment with As-IV may promote mitochondrial biogenesis and autophagy.

Parkin, from the cytosol, eliminates impaired mitochondria by targeted mitophagy (42). Additionally, parkin-induced mitochondrial fission, which requires Drp1, appears to be necessary for mitophagy (42-44). In the present study, treatment with Ang II increased the protein expression of parkin and Drp1, and As-IV increased the protein expression of parkin and Drp1 (Fig. 7A). These findings suggest that As-IV promotes the autophagy of impaired mitochondria. A previous study suggested that increased ROS levels in the mitochondrial matrix result in mitochondrial damage and the subsequent activation of parkin-dependent mitophagy (45). Taken together with the results of our study, these findings suggest that As-IV promotes the autophagy of impaired mitochondria.

PGC-1 $\alpha$ serves as a central transcriptional control of mitochondrial biogenesis and respiratory function that coordinately controls the mitochondrial energy-generating functions in 
accordance with the metabolic demands forced by changing physiological conditions, aging and disease (46). PGC-1 $\alpha$ activates the expression of Tfam, and then the transcription and replication of mtDNA is directly activated by the translocation of Tfam into the mitochondria (47). In the present study, As-IV markedly enhanced the mRNA expression of PGC-1 $\alpha$ and Tfam (Fig. 7B), and the protein expression of PGC-1 $\alpha$ (Fig. 7C). These results may also explain the stimulative effect of As-IV on the levels of mtDNA (Fig. 5). In this study, we found no significant difference in the protein expression of PGC- $1 \alpha$ and the mRNA expression of Tfam between the untreated VSMCs and Ang II-treated VSMCs. A previous study suggested that Ang II is unable to lead to changes in the mRNA expression of PGC-1 $\alpha$ and Tfam (48). Taken together with the results of our study, these findings suggest that As-IV enhances the expression of PGC-1 $\alpha$ and Tfam and promotes mitochondrial biogenesis.

In conclusion, this study clearly demonstrates that As-IV reverses the structural and biochemical abnormalities caused by Ang II in VSMCs, by modulating mtROS production and eliminating mtROS through its antioxidant effects, as well as by promoting mitochondrial autophagy and mitochondrial biogenesis. These findings suggest that mitochondria-targeted antioxidants may represent an attractive novel strategy for the treatment of a number of pathological conditions caused by the Ang II-mediated production of mtROS.

\section{Acknowledgements}

The present study was funded by grants from the National Natural Science Foundation of China (nos. 81170220 and 81100156), the Jiangsu Province Health Foundation (RC2011075), the Priority Academic Program Development of Jiangsu Higher Education Institutions and the Jiangsu Province Science and Technology Support Program (BE2011803).

\section{References}

1. Yu E, Calvert PA, Mercer JR, Harrison J, Baker L, Figg NL, Kumar S, Wang JC, Hurst LA, Obaid DR, et al: Mitochondrial DNA damage can promote atherosclerosis independently of reactive oxygen species through effects on smooth muscle cells and monocytes and correlates with higher-risk plaques in humans. Circulation 128: 702-712, 2013.

2. Mercer JR: Mitochondrial bioenergetics and therapeutic intervention in cardiovascular disease. Pharmacol Ther 141: 13-20, 2014.

3. Dikalov SI and Nazarewicz RR: Angiotensin II-induced production of mitochondrial reactive oxygen species: potential mechanisms and relevance for cardiovascular disease. Antioxid Redox Signal 19: 1085-1094, 2013.

4. Queliconi BB, Wojtovich AP, Nadtochiy SM, Kowaltowski AJ and Brookes PS: Redox regulation of the mitochondrial K(ATP) channel in cardioprotection. Biochim Biophys Acta 1813: 1309-1315, 2011.

5. Dikalov S: Cross talk between mitochondria and NADPH oxidases. Free Radic Biol Med 51: 1289-1301, 2011.

6. Doughan AK, Harrison DG and Dikalov SI: Molecular mechanisms of angiotensin II-mediated mitochondrial dysfunction: linking mitochondrial oxidative damage and vascular endothelial dysfunction. Circ Res 102: 488-496, 2008.

7. Wang H, Zhang Y, Xia T, Wei W, Chen F, Guo X and Li X: Synergistic promotion of blood vessel regeneration by astragaloside IV and ferulic acid from electrospun fibrous mats. Mol Pharm 10: 2394-2403, 2013.

8. Ko JK, Lam FY and Cheung AP: Amelioration of experimental colitis by Astragalus membranaceus through anti-oxidation and inhibition of adhesion molecule synthesis. World J Gastroenterol 11: 5787-5794, 2005.
9. Yang J, Wang HX, Zhang YJ, Yang YH, Lu ML, Zhang J, Li ST, Zhang SP and Li G: Astragaloside IV attenuates inflammatory cytokines by inhibiting TLR4/NF- $\kappa \mathrm{B}$ signaling pathway in isoproterenol-induced myocardial hypertrophy. J Ethnopharmacol 150: 1062-1070, 2013.

10. Gui D, Huang J, Guo Y, Chen J, Chen Y, Xiao W, Liu X and Wang N: Astragaloside IV ameliorates renal injury in streptozotocin-induced diabetic rats through inhibiting NF- $\mathrm{kB}$-mediated inflammatory genes expression. Cytokine 61: 970-977, 2013.

11. Xu W, Shao X, Tian L, Gu L, Zhang M, Wang Q, Wu B, Wang L, Yao J, Xu X, et al: Astragaloside IV ameliorates renal fibrosis via the inhibition of mitogen-activated protein kinases and antiapoptosis in vivo and in vitro. J Pharmacol Exp Ther 350: 552-562, 2014

12. Wu X, Cao Y, Nie J, Liu H, Lu S, Hu X, Zhu J, Zhao X, Chen J, Chen $\mathrm{X}$, et al: Genetic and pharmacological inhibition of Rheb1-mTORC1 signaling exerts cardioprotection against adverse cardiac remodeling in mice. Am J Pathol 182: 2005-2014, 2013.

13. Zhang WD, Zhang C, Wang XH, Gao PJ, Zhu DL, Chen H, Liu RH and Li HL: Astragaloside IV dilates aortic vessels from normal and spontaneously hypertensive rats through endothelium-dependent and endothelium-independent ways. Planta Med 72: 621-626, 2006.

14. Yin Y, Qi F, Song Z, Zhang B and Teng J: Ferulic acid combined with astragaloside IV protects against vascular endothelial dysfunction in diabetic rats. Biosci Trends 8: 217-226, 2014.

15. Zhao Z, Wang W, Wang F, Zhao K, Han Y, Xu W and Tang L: Effects of Astragaloside IV on heart failure in rats. Chin Med 4: 6, 2009.

16. Xu XL, Ji H, Gu SY, Shao Q, Huang QJ and Cheng YP: Modification of alterations in cardiac function and sarcoplasmic reticulum by astragaloside IV in myocardial injury in vivo. Eur $\mathrm{J}$ Pharmacol 568: 203-212, 2007.

17. Qiu LH, Xie XJ and Zhang BQ: Astragaloside IV improves homocysteine-induced acute phase endothelial dysfunction via antioxidation. Biol Pharm Bull 33: 641-646, 2010.

18. Sun L, Li W, Li W, Xiong L, Li G and Ma R: Astragaloside IV prevents damage to human mesangial cells through the inhibition of the NADPH oxidase/ROS/Akt/NF- $\mathrm{KB}$ pathway under high glucose conditions. Int J Mol Med 34: 167-176, 2014

19. Sun Q, Jia N, Wang W, Jin H, Xu J and Hu H: Protective effects of astragaloside IV against amyloid beta1-42 neurotoxicity by inhibiting the mitochondrial permeability transition pore opening. PLoS One 9: e98866, 2014.

20. Hu JY, Han J, Chu ZG, Song HP, Zhang DX, Zhang Q and Huang YS: Astragaloside IV attenuates hypoxia-induced cardiomyocyte damage in rats by upregulating superoxide dismutase-1 levels. Clin Exp Pharmacol Physiol 36: 351-357, 2009.

21. Tu L, Pan CS, Wei XH, Yan L, Liu YY, Fan JY, Mu HN, Li Q, Li L, Zhang Y, et al: Astragaloside IV protects heart from ischemia and reperfusion injury via energy regulation mechanisms. Microcirculation 20: 736-747, 2013.

22. Shen Y, Tian Y, Yang J, Shi X, Ouyang L, Gao J and Lu J: Dual effects of carnosine on energy metabolism of cultured cortical astrocytes under normal and ischemic conditions. Regul Pept 192-193: 45-52, 2014.

23. Mukhopadhyay P, Rajesh M, Haskó G, Hawkins BJ, Madesh M and Pacher P: Simultaneous detection of apoptosis and mitochondrial superoxide production in live cells by flow cytometry and confocal microscopy. Nat Protoc 2: 2295-2301, 2007.

24. Laurindo FR, de Souza HP, Pedro MA and Janiszewski M: Redox aspects of vascular response to injury. Methods Enzymol 352: 432-454, 2002.

25. Herst PM and Berridge MV: Cell surface oxygen consumption: a major contributor to cellular oxygen consumption in glycolytic cancer cell lines. Biochim Biophys Acta 1767: 170-177, 2007.

26. Xie H, Lev D, Gong Y, Wang S, Pollock RE, Wu X and Gu J: Reduced mitochondrial DNA copy number in peripheral blood leukocytes increases the risk of soft tissue sarcoma. Carcinogenesis 34: 1039-1043, 2013.

27. Elahi MM, Kong YX and Matata BM: Oxidative stress as a mediator of cardiovascular disease. Oxid Med Cell Longev 2: 259-269, 2009.

28. Wei Y, Clark SE, Thyfault JP, Uptergrove GM, Li W, Whaley-Connell AT, Ferrario CM, Sowers JR and Ibdah JA: Oxidative stress-mediated mitochondrial dysfunction contributes to angiotensin II-induced nonalcoholic fatty liver disease in transgenic Ren2 rats. Am J Pathol 174: 1329-1337, 2009.

29. Prathapan A, Vineetha VP and Raghu KG: Protective effect of Boerhaavia diffusa L. against mitochondrial dysfunction in angiotensin II induced hypertrophy in $\mathrm{H} 9 \mathrm{c} 2$ cardiomyoblast cells. PLoS One 9: e96220, 2014. 
30. Schulz E, Wenzel P, Munzel T and Daiber A: Mitochondrial redox signaling: Interaction of mitochondrial reactive oxygen species with other sources of oxidative stress. Antioxid Redox Signal 20: 308-324, 2014

31. Lijnen PJ, van Pelt JF and Fagard RH: Downregulation of manganese superoxide dismutase by angiotensin II in cardiac fibroblasts of rats: Association with oxidative stress in myocardium. Am J Hypertens 23: 1128-1135, 2010.

32. Touyz RM: Reactive oxygen species and angiotensin II signaling in vascular cells - implications in cardiovascular disease. Braz J Med Biol Res 37: 1263-1273, 2004.

33. Kimura S, Zhang GX, Nishiyama A, Shokoji T, Yao L, Fan YY, Rahman M and Abe Y: Mitochondria-derived reactive oxygen species and vascular MAP kinases: comparison of angiotensin II and diazoxide. Hypertension 45: 438-444, 2005.

34. Zablocki D and Sadoshima J: Angiotensin II and oxidative stress in the failing heart. Antioxid Redox Signal19: 1095-1109, 2013.

35. Bedard K and Krause KH: The NOX family of ROS-generating NADPH oxidases: physiology and pathophysiology. Physiol Rev 87: 245-313, 2007.

36. Suematsu N, Tsutsui H, Wen J, Kang D, Ikeuchi M, Ide T, Hayashidani S, Shiomi T, Kubota T, Hamasaki N and Takeshita A: Oxidative stress mediates tumor necrosis factor-alpha-induced mitochondrial DNA damage and dysfunction in cardiac myocytes. Circulation 107: 1418-1423, 2003.

37. Li H, Horke S and Förstermann U: Oxidative stress in vascular disease and its pharmacological prevention. Trends Pharmacol Sci 34: 313-319, 2013.

38. Qi W, Niu J, Qin Q, Qiao Z and Gu Y: Astragaloside IV attenuates glycated albumin-induced epithelial-to-mesenchymal transition by inhibiting oxidative stress in renal proximal tubular cells. Cell Stress Chaperones 19: 105-114, 2014.

39. Wang YY, Peng Y, Zhang Q, Wu YN, Song JQ and Liu YX: Effect of ERK1/2 signaling pathway on astragaloside IV protects $\mathrm{H} 9 \mathrm{c} 2$ cells against $\mathrm{H}_{2} \mathrm{O}_{2}$-induced oxidative injury. Zhongguo Ying Yong Sheng Li Xue Za Zh27: 363-367, 2011 (In Chinese).
40. Mammucari $\mathrm{C}$ and Rizzuto R: Signaling pathways in mitochondrial dysfunction and aging. Mech Ageing Dev 131: 536-543, 2010.

41. Lee J, Giordano S and Zhang J: Autophagy, mitochondria and oxidative stress: cross-talk and redox signalling. Biochem J 441: 523-540, 2012.

42. Narendra DP and Youle RJ: Targeting mitochondrial dysfunction: role for PINK1 and Parkin in mitochondrial quality control. Antioxid Redox Signal 14: 1929-1938, 2011.

43. Kageyama Y, Hoshijima M, Seo K, Bedja D, Sysa-Shah P, Andrabi SA, Chen W, Höke A, Dawson VL, Dawson TM, et al: Parkin-independent mitophagy requires Drpl and maintains the integrity of mammalian heart and brain. EMBO J 33: 2798-2813, 2014.

44. Deng H, Dodson MW, Huang H and Guo M: The Parkinson's disease genes pink1 and parkin promote mitochondrial fission and/or inhibit fusion in Drosophila. Proc Natl Acad Sci USA 105: 14503-14508, 2008.

45. Wang Y, Nartiss Y, Steipe B, McQuibban GA and Kim PK: ROS-induced mitochondrial depolarization initiates PARK2/PARKIN-dependent mitochondrial degradation by autophagy. Autophagy 8: 1462-1476, 2012.

46. Scarpulla RC, Vega RB and Kelly DP: Transcriptional integration of mitochondrial biogenesis. Trends Endocrinol Metab 23: 459-466, 2012.

47. Wu Z, Puigserver P, Andersson U, Zhang C, Adelmant G, Mootha V, Troy A, Cinti S, Lowell B, Scarpulla RC and Spiegelman BM: Mechanisms controlling mitochondrial biogenesis and respiration through the thermogenic coactivator PGC-1. Cell 98: 115-124, 1999.

48. Biala A, Tauriainen E, Siltanen A, Shi J, Merasto S, Louhelainen M, Martonen E, Finckenberg P, Muller DN and Mervaala E: Resveratrol induces mitochondrial biogenesis and ameliorates Ang II-induced cardiac remodeling in transgenic rats harboring human renin and angiotensinogen genes. Blood Press 19: 196-205, 2010 